Revue

de Sémantique

et Pragmatique
Revue de Sémantique et Pragmatique

35-36 | 2015

Interface sémantique/morphologie

\title{
Quand le cinétisme culturel provoque une énucléation partielle de la signification lexicale
}

When cultural kinesis causes a partial lexical meaning enucleation

ROCHAIX Valérie

\section{(2) OpenEdition}

Journals

Édition électronique

URL : http://journals.openedition.org/rsp/1676

DOI : $10.4000 /$ rsp. 1676

ISSN : 2610-4377

Éditeur

Presses universitaires d'Orléans

\section{Édition imprimée}

Date de publication : 1 mars 2015

Pagination : 211-226

ISSN : 1285-4093

\section{Référence électronique}

ROCHAIX Valérie, «Quand le cinétisme culturel provoque une énucléation partielle de la signification lexicale », Revue de Sémantique et Pragmatique [En ligne], 35-36 | 2015, mis en ligne le 01 mars 2016, consulté le 09 mai 2020. URL : http://journals.openedition.org/rsp/1676 ; DOI : https://doi.org/ $10.4000 /$ rsp. 1676 


\title{
QUAND LE CINÉTISME CULTUREL PROVOQUE UNE ÉNUCLÉATION PARTIELLE DE LA SIGNIFICATION LEXICALE
}

\author{
ROCHAIX Valérie \\ CoDiRe - IRFFLE, Université de Nantes
}

Les représentations de la signification lexicale de couteau, église, château ou carte postale n'ont a priori rien de commun. Et pourtant, si un locuteur associe dans son discours ces objets au lexème patrimoine, ces derniers semblent libérés de leurs fonctions religieuse, politique ou sociale (Malraux, 1947) pour être investis d'une nouvelle fonction, avec comme corollaire une modification de leur signification. Ce mécanisme est étudié et reconnu de longue date par les sciences humaines. Ainsi, le fait d'intégrer un objet à la sphère du patrimoine - c'est à dire décréter sa patrimonialisation - résulte, pour le sociologue Desvallées, "d'interventions et de stratégies concertées de marquage et de signalisation » (2011 : 423). Pour Davallon ou Gellereau, la « construction discursive » de l'objet patrimonial le déplace dans la sphère représentationnelle du sacré.

L'analyse linguistique peut en préciser le mécanisme. Du point de vue sémantique, cela peut se traduire par le fait que l'association de l'une ou l'autre désignation au mot patrimoine dans un énoncé manifeste d'une force locutoire spécifique de création de lien de dénomination. Notre hypothèse est que ce lien de dénomination est accompagné d'un mécanisme de « contamination discursive » du patrimoine sur un objet qu'il désigne, c'est à dire de l'inscription discursive d'associations argumentatives nouvelles issues de la signification lexicale de patrimoine dans la signification lexicale de cet objet. Le cinétisme s'exerçant aux dépens des traits essentiels de la signification initiale de ce dernier.

L'objet de cet article est de tester cette hypothèse à partir de l'analyse 
comparative des propriétés sémantiques d'un mot avant et après la « construction discursive » de la patrimonialisation. Elle se fonde sur une étude de cas menée dans une logique binaire de différenciation auprès de dix informateurs sur la représentation du mot couteau comme désignant un objet d'abord dans un contexte fonctionnel puis dans un contexte patrimonial. Exploratoire, elle ne prétend pas identifier les implications sémantiques de tout discours patrimonial. A partir d'un objet choisi parmi d'autres, nous entendons néanmoins affirmer la vraisemblance de notre hypothèse et d'autre part suggérer la localisation et l'ampleur de cette contamination.

Pour cela, nous nous appuierons sur le cadre théorique de description de la signification de la Sémantique des Possibles Argumentatifs [désormais SPA] (Galatanu, 2004, 2007, 2009), qui a pour originalité de formuler un point de contact entre la dimension conceptuelle du sens (la signification dans la langue) et les observables (dans la parole) et de distinguer les parties stables de la signification des parties plus évolutives, fortement impliquées dans le cinétisme de la signification qui nous préoccupe ici.

\section{PATRIMOINE. LES VALEURS PRÉSENTES AU STADE LEXICAL ANNONCIATRICES D'UNE FORCE ILLOCUTOIRE EN CONTEXTE}

À partir de l'étude de discours tenus par différents locuteurs sur des objets qu'ils considèrent comme faisant partie de leur patrimoine, Paveau observe une « nature fondamentalement sémiotique » du patrimoine (2009, 2011). Elle définit ce dernier comme « un dispositif social et un discours organisateur de la mémoire d'un groupe, dans un contexte historique et social donné (...), un ensemble d'images et de représentations, de décisions terminologiques et juridiques » (Paveau, 2009 : 36). Comme elle, nous nous proposons d'aborder patrimoine comme une construction mentale et discursive mais en rendant compte du potentiel discursif des entités linguistiques mobilisées. Et ce, dans une démarche d'Analyse linguistique du discours (Galatanu, 2009) conçue comme le repérage et l'étude des mécanismes sémantico-discursifs et pragmaticodiscursifs de production et d'interprétation du sens, situés au croisement de l'Analyse du Discours et de la Sémantique Lexicale.

\subsection{QUAND DIRE PATRIMOINE, C'EST FAIRE PATRIMOINE}

«Au seuil du XXI ${ }^{\mathrm{e}}$ siècle, le patrimoine est virtuellement devenu tout ce qu'un groupe décide d'intituler de la sorte » (Desvallées, $2011: 437$ ). Autrement dit, l'objet est « dit » patrimoine alors il est « fait » patrimoine. Celui-ci se trouverait par conséquent investi des propriétés du mot. Pour expliciter la capacité intrinsèque de patrimoine à modifier les propriétés sémantiques du mot auquel il est associé, nous nous référons à l'assertion d'Austin " to do something is in the full normal sense to do something» (Austin, 1962 [1970] : 109). Etablie 
dans le cadre d'une recherche menée entre énoncés performatifs et énoncés constatifs et constituant la base de sa théorie des actes de langage pour décrire les énoncés destinés à transformer la réalité, celle-ci traduit la force locutoire de l'entité. On peut donc supposer un caractère performatif déclaratif intrinsèque à patrimoine qui instaure, par sa convocation une nouvelle représentation du monde.

$\mathrm{Si}$, lorsqu'il patrimonialise un objet, c'est-à-dire qu'il le rend patrimonial, un locuteur assortit sa signification des valeurs du patrimoine, on peut alors émettre deux hypothèses : d'une part, un objet auquel on attribue des valeurs distinctes selon la relation que l'on entretient avec lui acquiert, lors de ce processus, un statut plus conventionnel, aspect explorable en relation avec la théorie des actes de langage succinctement abordé ci-dessus. D'autre part, quelles que soient leurs propriétés sémantiques initiales, les objets patrimoniaux sont porteurs des mêmes valeurs, celles du patrimoine, qui dépassent leurs attributs individuels. Nous laissons la première de côté. Une étude de cas a en revanche été menée pour mettre la seconde à l'épreuve, en testant le processus de contamination discursive liée au contexte, à l'aide d'une mise en scène suggérant un phénomène de patrimonialisation.

\subsection{LA SIGNIFICATION LEXICALE DE PATRIMOINE DANS LE CADRE DE LA SÉMANTIQUE DES POSSIBLES ARGUMENTATIFS}

Si l'on se place non pas dans une logique strictement référentialiste, qui renvoie à une description des conditions d'application ou de vérité, mais dans un paradigme enrichi de l'hypothèse du statut argumentatif du sens tel que développé par l'Argumentation dans la Langue (Anscombre, Ducrot, 1983), la description de la signification prend en compte, au-delà des traits référentiels (traits de prototype, Kleiber, 1990), des traits instructionnels au travers «des mécanismes dynamiques (déictiques, inférentiels), qui ne constituent pas de propriétés du référent, mais des balises plus ou moins rigides pour y arriver» (Kleiber, 1999 : 50). Ces derniers, relevant de la connotation, sont argumentatifs notamment. C'est dans ce paradigme que se situe la Sémantique des Possibles Argumentatifs (Galatanu, 2004, 2007, 2009).

D'inspiration putnamienne, ce modèle de description sémantique est conçu comme une approche lexicale holistique (puisque qu'elle regroupe différents types d'information classés selon leur centralité et leur pertinence et que le lexème est traité dans sa totalité), associative (reprenant la Théorie des Topoï (Anscombre, 1995), il permet d'étudier le caractère intrinsèque du lexème associé à d'autres unités sémantiques) et, enfin, encyclopédique (l'ensemble des connaissances sur l'entité en jeu contribuant au sens de l'expression que la désigne) de la signification. Dans la filiation de la sémantique argumentative, 
la SPA postule que 1) le potentiel axiologique (donc argumentatif) de la signification d'une entité lexicale s'inscrit et se réinscrit dans chaque occurrence discursive 2) ce potentiel (formulé en termes d'associations de blocs sémantiques d'argumentation) est ancré culturellement et donc évolutif 3) le langage est appréhendé comme outil cognitif de la modélisation du monde, la dimension argumentative se manifeste à travers l'association des propriétés essentielles avec les représentations associées durablement au mot et au niveau des séquences discursives potentielles. A partir de ces trois postulats, la SPA propose un outil d'analyse en 4 strates : le noyau (Putnam, 1975, 1990, 1994) et les stéréotypes (associés durablement au mot), une dénomination commune à Fradin, Putnam, Anscombre et Galatanu. La SPA propose de mettre en exergue les «possibles argumentatifs »(PA), des séquences discursives argumentatives potentielles permettant de prédire la possibilité de séquences discursives « réelles » et enfin, les « déploiements argumentatifs », dans le discours. Cette représentation sémantique entend ainsi rendre compte : 1) de la dimension descriptive de la signification, permettant de stabiliser le monde par la dimension langagière 2) de la partie «stable » de la signification et de sa partie évolutive, que le discours proposé charge et décharge de valeurs 3) du potentiel discursif (argumentatif) de la signification lexicale et 4) du potentiel cinétique du dispositif noyau-stéréotypes (Galatanu, 1999 : 48)

La SPA propose donc un modèle de description de la signification lexicale susceptible de rendre compte de ce que Kleiber appelle les représentations du monde « perçu » et « modélisé » par la langue. Elle insiste sur la dimension argumentative de la signification lexicale et propose un modèle pour la représenter.

\section{3. LA DESCRIPTION DE LA SIGNIFICATION LEXICALE DE PATRIMOINE}

Apparu au XII ${ }^{\mathrm{e}}$ siècle dans le vocabulaire juridique, le mot patrimoine est encore défini à l'aube de la Révolution française comme « le bien qui vient du père et de la mère, que l'on a hérité du père et de la mère » ainsi que « figurément, une chose qui est le revenu ordinaire et naturel d'un homme $»^{1}$. A partir de 1823 , on le trouve sous son acception quasi-contemporaine, à savoir «ce qui est transmis à une personne, une collectivité, par les ancêtres, les générations précédentes, et qui est considéré comme un héritage commun $»^{2}$. L'analyse de sa définition dans plusieurs dictionnaires actuels ${ }^{3}$ (enrichie des enchaînements

1 Dictionnaire de l'Académie Française, Classique Garnier [édition électronique], $1^{\mathrm{e}}$ éd. $1694,5^{\mathrm{e}}$ éd. 1785

2 Nouveau dictionnaire de langue française Laveaux, 1823, Paris

3 Description établie à partir des références suivantes : le dictionnaire de l'Académie Française (1789) pour le XVIII ${ }^{\mathrm{e}}$ s. ; le dictionnaire général pour la maitrise de la langue française, la culture classique et la langue contemporaine pour le français moderne et 
discursifs présentées pour l'illustrer) suggère la représentation suivante, sachant que « partant du principe que la signification lexicale du mot est potentiellement argumentative (Galatanu, 2008), des connecteurs [expriment] les enchaînements argumentatifs normatifs (donc) ou transgressifs (pourtant) $»^{4}$ :

\begin{tabular}{|c|c|c|}
\hline $\begin{array}{l}\text { Noyau }(\mathbf{N}) \\
\text { (propriétés } \\
\text { essentielles } \\
\text { de } \\
\text { patrimoine) }\end{array}$ & $\begin{array}{l}\text { Stéréotypes (Sts) } \\
\text { (ensemble ouvert (et moins } \\
\text { stable) d'associations des } \\
\text { éléments du noyau avec d'autres } \\
\text { représentations constituants des } \\
\text { blocs d'argumentation internes) } \\
\end{array}$ & $\begin{array}{l}\text { Possibles argumentatifs (PA) } \\
\text { (associations de patrimoine avec les } \\
\text { éléments de son stéréotype) }\end{array}$ \\
\hline héritage & $\begin{array}{l}\text { être inscrit dans une lignée } \\
\rightarrow \text { avoir une ascendance / } \\
\quad \text { descendance } \\
\rightarrow \text { avoir des racines } \\
\rightarrow \text { avoir une histoire } \\
\text { être légitime } \\
\rightarrow \text { accepter / recevoir } \\
\rightarrow \text { avoir des droits } \\
\rightarrow \text { être privilégié }\end{array}$ & $\begin{array}{l}\text { patrimoine donc être inscrit dans une lignée } \\
\text { patrimoine dc avoir une ascendance /desc. } \\
\text { patrimoine dc avoir des racines } \\
\text { patrimoine dc avoir une histoire } \\
\text { patrimoine dc être légitime } \\
\text { patrimoine dc accepter /recevoir } \\
\text { patrimoine dc avoir des droits } \\
\text { patrimoine dc être privilégié }\end{array}$ \\
\hline avoir & $\begin{array}{l}\text { avoirs } \\
\rightarrow \text { avoir des droits } \\
\rightarrow \text { avoir une sécurité } \\
\rightarrow \text { avoir une responsabilité } \\
\rightarrow \text { avoir des contraintes } \\
\text { richesse } \\
\rightarrow \text { avoir des biens matériels ou } \\
\quad \text { immat. } \\
\rightarrow \text { avec ou sans valeur comptable } \\
\rightarrow \text { avoir un privilège } \\
\rightarrow \text { être supérieur }\end{array}$ & $\begin{array}{l}\text { patrimoine donc avoir des droits } \\
\text { patrimoine dc avoir une sécurité } \\
\text { patrimoine dc avoir une responsabilité } \\
\text { patrimoine dc avoir des contraintes } \\
\\
\text { patrimoine dc avoir des biens matériels ou } \\
\quad \text { immatériels } \\
\text { patrimoine dc être riche (avec ou sans } \\
\quad \text { valeur comptable) } \\
\text { patrimoine dc avoir un privilège } \\
\text { patrimoine dc être supérieur }\end{array}$ \\
\hline transmission & $\begin{array}{l}\text { s'inscrire dans la durée } \\
\rightarrow \text { conserver } \\
\rightarrow \text { valoriser } \\
\rightarrow \text { avoir honte si dilapidé } \\
\rightarrow \text { lien }\end{array}$ & $\begin{array}{l}\text { patrimoine donc être inscrit dans la durée } \\
\text { patrimoine dc conserver } \\
\text { patrimoine dc valoriser } \\
\text { patrimoine dc honte si dilapidé } \\
\text { patrimoine dc lien }\end{array}$ \\
\hline valeur & $\begin{array}{l}\text { axiologique } \\
\text { aléthique }\end{array}$ & positive \\
\hline
\end{tabular}

figure 1 : Description de la représentation sémantique de patrimoine à partir $d u$ modèle de la SPA (Galatanu, 2004, 2007, 2009).

contemporain ainsi que le Grand Robert de la langue contemporaine, le Dictionnaire Grand Larousse universel, le Dictionnaire étymologique et historique du français, le dictionnaire Le Robert de la langue française, le Lexis, le Grand Littré et le Trésor de la langue française

${ }^{4}$ Bellachhab A., Galatanu O. (2012) La violence verbale : représentation sémantique, typologie et mécanismes discursifs. Signes, Discours et Sociétés [en ligne] URL : www. revue-signes.info/document.php?id=2893.ISSN 
Le dispositif signifiant de patrimoine propose une représentation conceptuelle du mot. La dernière colonne est à considérer comme une interface entre langue et parole, signification et sens qui permet de prévoir les déploiements possibles dans le discours patrimonial.

Il fait apparaître 3 éléments nucléaires essentiels à la définition du mot qui, en l'occurrence, renvoie à l'étymologie du mot, à savoir sa construction à partir du latin patrimonium dérivée de pater. Des stéréotypes de patrimoine inscrivent au niveau de sa signification lexicale des éléments tels que "valeur », «inscription dans la durée » et «inscription dans un lignage » qui impliquent des possibilités d'orientation argumentative dans une dimension axiologiquement positive.

\section{UNE EXPÉRIMENTATION DE CETTE FORCE EN CONTEXTE : COUTEAU, OBJET FONCTIONNEL ET COUTEAU, OBJET PATRIMONIAL}

Pour tester l'évolution sémantique d'un mot désignant un objet au contact des représentations mentales (Searle) de patrimoine, nous proposons l'étude d'un mot renvoyant, en contexte, à un objet. Sans ignorer les travaux sur la multiplicité des représentations inhérentes à tout objet au-delà de ses fonctions concrètes ou symboliques (Baudrillard, Boudon, Glassie et Moles), nous choisirons de nous en tenir à une approche binaire, à savoir objet-fonction concrète fonctionnelle et objet-fonction symbolique patrimoniale (à l'issue d'une remédiation) et de l'exercer sur un objet fréquent aussi bien dans un contexte très fonctionnel que dans des mises en scène patrimoniales. Du point de vue sémantique, il s'agira d'un mot se situant au niveau de base (Kleiber, 1993) dont la représentation mentale n'est pas trop formelle (Searle, Putnam), sans prise en compte de constructions taxinomiques relatives à des notions de styles ou d'époques. Le mot couteau répond à ces critères : il sert à désigner une catégorie d'outils ou d'armes ayant un nombre important de représentants, dont la connaissance est largement partagée à travers les cultures, le temps et l'espace. Sa convocation est très courante dans la vie quotidienne comme dans des discours patrimoniaux. 


\begin{tabular}{|c|c|c|}
\hline Noyau & Stéréotypes & Possibles argumentatifs \\
\hline $\begin{array}{l}\text { lame + } \\
\text { manche } \\
\text { dc }\end{array}$ & $\begin{array}{l}\text { diversité des matériaux } \\
\text { forme spécifique } \\
\text { long } \\
\text { droit } \\
\text { assemblage } \\
\text { pliable ou non } \\
\text { simple ou double lame }\end{array}$ & $\begin{array}{l}\text { couteau donc diversité des matériaux } \\
\text { couteau dc forme spécifique } \\
\text { couteau dc long } \\
\text { couteau dc droit } \\
\text { couteau dc assemblage } \\
\text { couteau dc pliable/non pliable } \\
\text { couteau dc simple/double lame }\end{array}$ \\
\hline $\begin{array}{l}\text { couper } \\
\mathrm{dc}\end{array}$ & $\begin{array}{l}\rightarrow \text { couper } \rightarrow \text { pouvoir blesser } \\
\rightarrow \text { pouvoir faire/avoir mal } \\
\rightarrow \text { trancher } \\
\rightarrow \text { séparer } \\
\rightarrow \text { menacer } \\
\rightarrow \text { représenter un danger }\end{array}$ & $\begin{array}{l}\text { couteau donc pouvoir blesser } \\
\text { couteau dc pouvoir/faire mal } \\
\text { couteau dc trancher } \\
\text { couteau dc séparer } \\
\text { couteau dc menacer } \\
\text { couteau dc représenter un danger }\end{array}$ \\
\hline instrument & $\begin{array}{l}\rightarrow \text { pratique/utile pour } \\
\text {. couper } \\
\text {. cuisiner } \\
\text {.manger } \\
\text {. nettoyer } \\
\text {. gratter } \\
\text {. raser } \\
\text {. étaler } \\
\rightarrow \text { chasser, combattre } \\
\rightarrow \text { permettre geste précis, net }\end{array}$ & $\begin{array}{l}\text { couteau donc pratique/utile } \\
\text { couteau dc couper } \\
\text { couteau dc cuisiner } \\
\text { couteau dc manger } \\
\text { couteau dc nettoyer } \\
\text { couteau dc gratter } \\
\text { couteau dc raser } \\
\text { couteau dc étaler } \\
\text { couteau dc chasser/combattre } \\
\text { couteau dc geste précis/net }\end{array}$ \\
\hline
\end{tabular}

figure 2 : Description de la représentation sémantique de couteau à partir du modèle de la SPA (Galatanu, 2004, 2007, 2009); cf figure 1

\subsection{MÉTHODE}

Un questionnaire a été soumis en entretien à dix informateurs adultes francophones. Il comporte trois sections : dans une dynamique de différenciation, une première fiche concerne le mot couteau dans un contexte fonctionnel, celuici est suggéré par plusieurs photos de couteaux fonctionnels de types différents (batterie de cuisine et couteau de table standard, canif) afin d'éviter les risques d'énonciation des caractéristiques intrinsèques de chaque objet au profit d'une appréhension générique de l'objet. La deuxième section concerne le mot couteau, envisagé comme objet patrimonial, représenté en l'occurrence, par trois illustrations de couteaux derrière des vitrines (identifiées comme symbolisant la mise en scène patrimoniale; cf œuvre, vitrine de référence, Boltanski, 1971) extraites du site du musée de la coutellerie de Thiers (Puy-de-Dôme) ${ }^{5}$ et de l'illustration d'une exposition sur la coutellerie au musée des Meilleurs Ouvriers de France de Bourges (Cher) ${ }^{6}$. La proposition d'illustrations sans texte associé

\footnotetext{
5 www.auvergne-tourisme.info

6 www.encyclopedie.bourges.com
} 
a pour but de fournir une représentation de l'objet comme objet fonctionnel puis comme objet patrimonial tout en influençant au minimum les réponses des informateurs par un discours argumentatif qui l'accompagnerait. La troisième section concerne le mot patrimoine, sans illustration associée. Dans les trois questionnaires, les informateurs doivent dans un premier temps fournir des associations spontanées puis non-spontanées à chacun des objets.

\subsection{RÉSULTATS ET DISCUSSION}

2.1.1. Les associations spontanées varient selon l'environnement du couteau, comme le montrent les graphiques suivants :

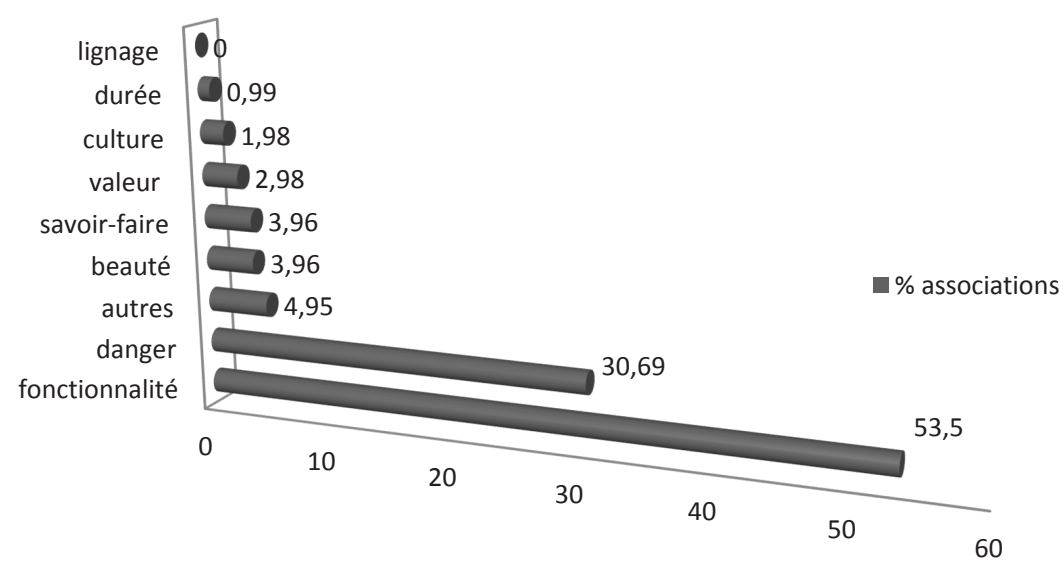

figure 3 : Répartition thématique des 100 (10x10) associations spontanées données à couteau

Ont été assemblés dans le groupe «fonctionnalité » les verbes d'action relatifs au repas (manger, cuisiner, trancher, hacher, éplucher...) ou au bricolage (réparer, bricoler...) et leurs substantifs ainsi que les adjectifs relatifs à la fonctionnalité (fonctionnel, pratique, efficace...) et les mots désignant des ingrédients (viande, huîtres, fromage...)

Ont été assemblés dans le groupe « danger » les substantifs relatifs à la menace (danger, menace, ...), au combat (tuer, crime, meurtre, armes, Rambo, défense...), à la douleur (sang, sparadrap...) et les adjectifs et les substantifs désignant des valeurs éthiques et morales négatives (malsain, méchanceté...)

Il apparaît que ces deux groupes dominent largement le champ des représentations relatives à couteau $(84,7 \%)$. Aucune autre dimension ne se distingue par ailleurs. 


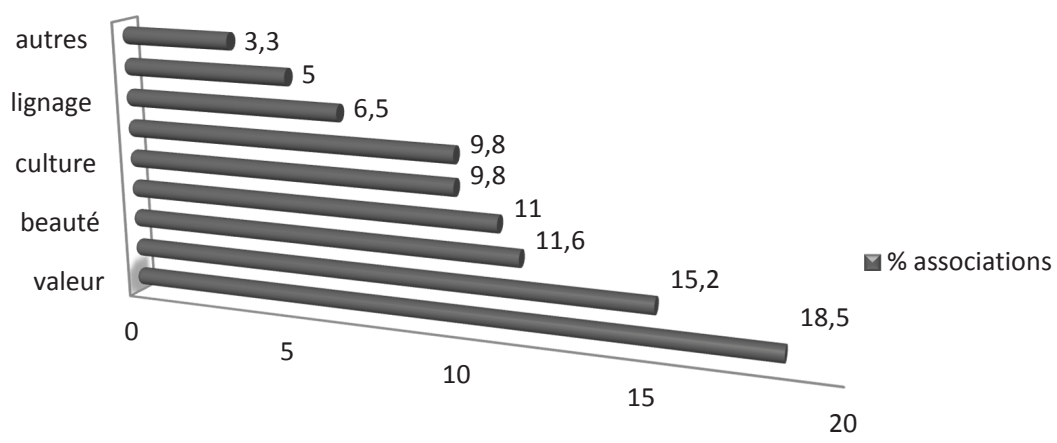

figure 4 : Répartition thématique des 100 associations spontanées données à couteau "exposé dans un musée »

Ont été intégrés dans le groupe «valeur », les substantifs relatifs à la valeur marchande (argent, richesse, préciosité) ou institutionnelle (prestige, pouvoir...). Dans le groupe « beauté » figurent l'adjectif beau(x) cités 6 fois ainsi que des substantifs et adjectifs liés à la mise en valeur (décoration, orné/ travaillé...). Dans le groupe « culture », on trouve les références à des cultures, des styles, des modes de vie.

On note que les substantifs relatifs à danger/menace et à la fonctionnalité ont quasiment disparu : seules deux associations (et donc 2\% des réponses) pouvant entrer dans le premier champ ont été spontanément citées une fois : « blessure », et « dangereux ». Celui de la fonctionnalité apparait également deux fois («manger» et « couper»)

Il apparaît donc que le co(n)texte influe bien sur la représentation du mot. Positionné dans un premier temps dans le champ des valeurs pragmatiques et matérialisé par une surreprésentation des verbes, adjectifs, le mot et l'objet qu'il désigne sont ensuite également associés à des valeurs plus esthétisantes. On observe que le recours aux verbes devient là quasi-inexistant.

L'informateur (le locuteur) opère une déconstruction du savoir proprement sémantique et reconstruit la signification du mot de façon contrainte par le contexte (la vitrine en l'occurrence). En insérant de nouveaux stéréotypes qui ne figurent pas dans la signification initiale, il invente un déploiement nonconformiste du potentiel discursif de la signification de couteau et opère donc effectivement un cinétisme de la signification lexicale. Il s'agit d'un mécanisme sémantico-discursif d'extension de stéréotypes et d'enrichissement de la signification. Celui-ci est acceptable pour qui admet que ce qui est dans un musée est sans conteste «patrimoine ».

Cet enrichissement de la signification de l'objet symbolique représente-til un apport brut ou des stéréotypes initialement contenus dans la signification concrète sont-ils masqués lors de cette opération? 


\subsubsection{Une contamination discursive partielle}

2.2.2.1. Les traits sémantiques de patrimoine contaminent couteau, objet patrimonial

Lors de cette même étude, un tableau d'associations non-spontanées à couteau et couteau « exposé dans un musée » (toujours introduits par les mêmes images) a été soumis aux informateurs. Il contient d'une part les traits sémantiques systématiquement associées à patrimoine dans une enquête précédemment menée auprès de 40 locuteurs français (à partir de la description en figure 1), à savoir richesse - lien affectif - transmission - ascendancel descendance - inscription dans la durée - art - beauté - bien matériel - histoire - héritage - tradition - racines - lien (inter)générationnel - lien familial mémoire - conservation - témoignage. Il reprend d'autre part les éléments de la description de la signification de couteau établie plus haut. Chacune des 10 personnes interrogées devaient cocher les propriétés essentielles de couteau dans chacun des deux cas. Leur réponse sont synthétisées dans le tableau suivant : en ordonnée, les propriétés proposées ; en abscisse, le nombre d'interlocuteurs ayant considéré ces associations comme toujours possibles à (et donc propriétés essentielles de) couteau envisagé comme fonctionnel (en gris) ou comme patrimonial (en noir).

Le schéma ci-dessus décrit :

- une forte distinction des représentations ou images mentales associées à l'objet en fonction du contexte.

- les éléments étant systématiquement associés à couteau : objet long, pratique, avec une lame (et donc pointu/piquant), pour cuisiner/manger, trancher/couper.

- les éléments étant systématiquement associés à couteau (dans un musée) : richesse, inscription dans la durée, art, beauté, histoire, héritage, mémoire, témoignage. Ces dernières valeurs sont présentes dans la signification lexicale de patrimoine issue de l'enquête sémantique.

Il en ressort qu'un seul élément est associé systématiquement dans les deux cas : la lame. Elle est cependant comme « dépossédée » de sa fonction originelle qui est de couper (mais dans une moindre mesure de trancher/séparer) pour le couteau patrimonialisé.

À condition que notre hypothèse des constituants des noyaux de chacun des éléments étudiés soit correcte, la création du lien de dénomination de l'objet patrimonial agit non seulement sur les stéréotypes mais aussi sur les traits essentiels de couteau. En effet, ses composantes fonctionnelles sont soit amoindries (pour séparer/tranchant), soit ignorées (pour couper et pratique). 


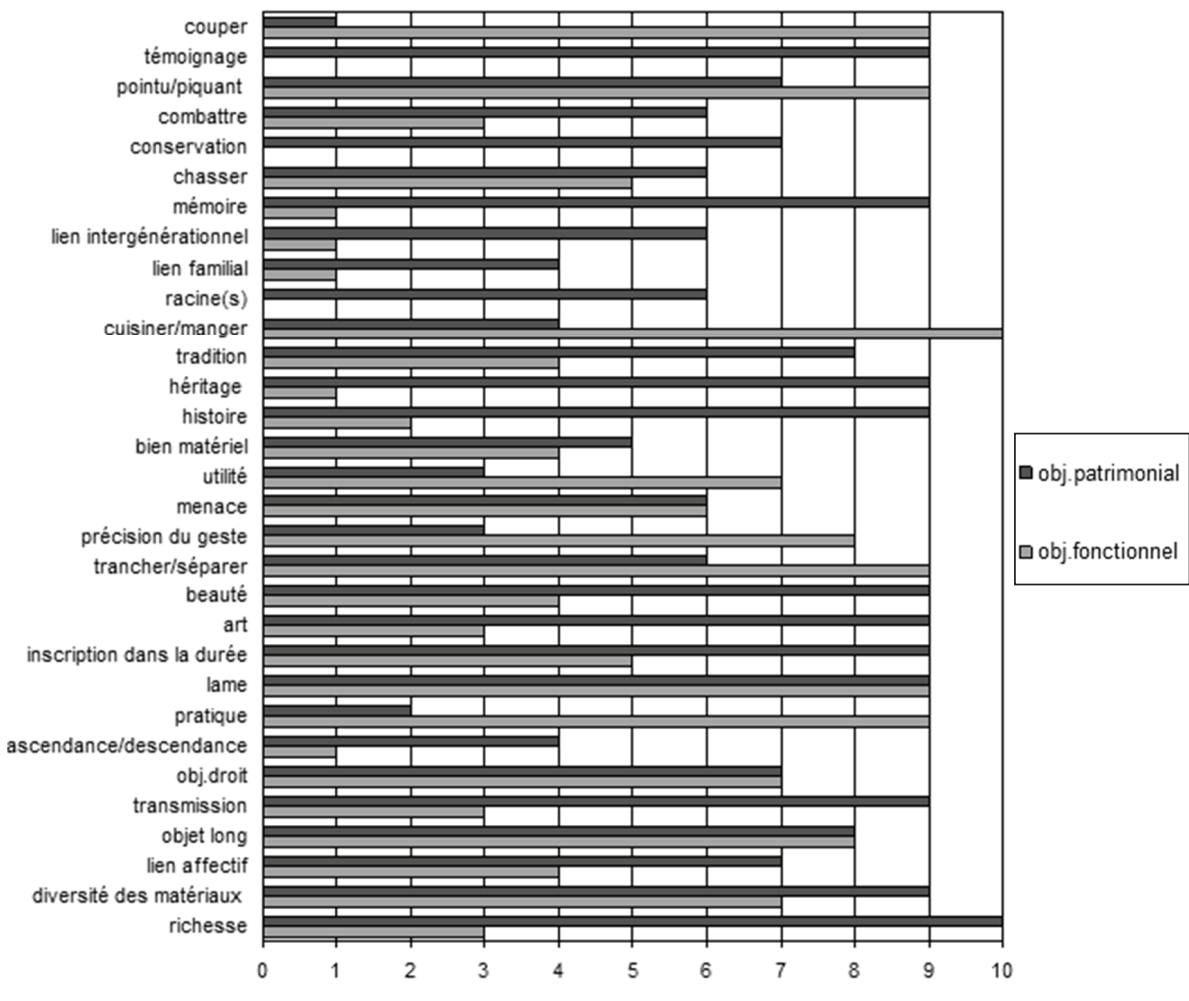

figure 5 : diagramme comparatif des associations considérées comme des éléments essentiels (toujours associées) avec couteau (objet fonctionnel) et couteau exposé dans un musée (objet patrimonial)

2.2.2.2 Pas de désactivation, mais une « déréalisation »?

Cette disparition apparente de certains traits sémantiques dans l'étude de cas peut-elle être interprétée comme la marque d'une possible désactivation complète de traits a priori essentiels en vertu d'un processus de contamination discursive ? Pour tester s'il s'agit de traits ignorés par les informateurs ou désactivés par leur nouveau schéma représentationnel, nous proposons d'effectuer des opérations de commutation des connecteurs logiques qui relient certaines associations constitutives du noyau :

(i) Le couteau ne coupe pas et il est précieux car c'est mon patrimoine

(ii) Le couteau ne coupe pas pourtant il est précieux car c'est mon patrimoine 
Si l'association à une distance faible de couteau et patrimoine désactive le trait fonctionnel de couteau, à savoir couper, alors (i) est possible, car il ne représente pas de transgression par rapport à sa nouvelle signification lexicale. Si les traits fonctionnels du noyau sont juste « endormis », alors (ii) est plus certainement possible. Si couper est désactivé alors, (i) est possible. Or (i) semble difficilement acceptable. Le trait sémantique couper peut donc presque disparaître des éléments associés à couteau dans la vitrine sans pour autant que l'on puisse postuler une énucléation partielle de la signification lexicale.

Notre hypothèse est que patrimoine agit ici comme un modificateur des traits sémantiques de couteau. Développés par Ducrot (1995) pour qualifier les adjectifs ou les adverbes susceptibles d'accroître l'applicabilité de la signification lexicale, en l'occurrence, dans la perspective de la SPA, d'un stéréotype (modificateur réalisant) ou de l'abaisser (modificateur déréalisant), le concept est applicable au syntagme « exposé dans un musée » que l'on substitue à « patrimonial », très peu utilisé dans la langue commune. En effet, outre les éléments cités plus haut, l'analyse comparative montre par exemple que le couteau s'inscrit d'autant plus dans la durée qu'il est dans un contexte patrimonial. De même, le couteau est d'autant moins pratique qu'il est regardé dans ce même contexte. "Patrimoine /exposé dans un musée » influe donc sur « la force avec laquelle on applique, à propos d'un objet ou d'une notion, les topoï constituants sa signification » (Ducrot, 1995 : 145-146).

\section{LIMITES ET RESTRICTIONS}

\subsection{UNE PROBLÉMATIQUE INTERFÉRENCE PRAGMATIQUE DANS LA REPRÉSENTATION DE COUTEAU}

Le lexème couteau a été choisi en raison de critères sémantiques (niveau de catégorisation et image mentale précise mais non figée). Cependant, la dimension affective relative à certains objets auxquels il renvoie avait dans un premier temps été négligée. Ainsi, la présence d'un Opinel était apparue comme convoquant immédiatement la notion de patrimoine (transmission paternelle, liens familiaux lors de scènes de partage...) La possible interférence de propriétés telles que «l'inscription dans la durée » d'un objet en général solide et résistant et « tradition » pour un objet lié aux parures, à l'art guerrier et culinaire ont également été sous-estimées en avant-propos. Une enquête reposant sur davantage d'illustrations pourrait permettre de lever l'ambiguïté. Une rapide évaluation de ces critères nous a amené à retirer l'Opinel des images associées à couteau fonctionnel, rendu le plus neutre possible, et à traduire avec précaution les résultats de cette étude de cas. Il est également à noter que l'objet couteau est porteur de valeurs différentes en fonction des cultures dans lesquelles il est représenté et des langues dans lesquelles il est déployé (Mathieu, 1987 : 16). 


\subsection{LA CATÉGORIE GRAMMATICALE NON PRISE EN COMPTE}

Les associations fournies par les informateurs appartiennent à différentes catégories grammaticales, nominales, verbales ou adjectivales. Ces variations n'ont pas été prises en compte dans l'analyse qui a traité chaque forme comme ayant le même rôle informatif et argumentatif. Ce choix méthodologique repose sur une approche lexicale. Mais il est possible que la prise en compte de ces options puisse affiner l'analyse. On peut notamment se demander si trancher et tranchant impliquent la même visée argumentative, sans modification de polarité. Trancher n'implique-t-il pas, par exemple une visée axiologique pragmatique positive tandis que tranchant orienterait vers une dimension affective/hédonique négative?

\subsection{UN MÉCANISME GÉNÉRALISABLE ?}

Un objet en devenant objet patrimonial se dote des stéréotypes de patrimoine par un phénomène de contamination discursive. Par ailleurs, une partie de ses traits initiaux, y compris ceux faisant partie de son noyau est au moins ignorée. Cette observation du processus discursif subi par couteau est-elle généralisable à l'ensemble des désignations d'objets du patrimoine ?

Il ne s'agit pas de tester la signification lexicale de tous les objets patrimoniaux d'autant plus que la notion, impliquée dans des champs sociétaux de plus en plus divers, est devenue " englobante (...) visant [maintenant] l'ensemble de l'activité humaine en tout point et à toutes époques de l'humanité» (Leniaud, 2002 : 11), voire obsessionnelle (Derrida, 1995) jusqu'à concerner aussi bien la gastronomie que les romans noirs et les jeux vidéo.

On peut néanmoins expérimenter la force locutoire de patrimoine/ patrimonial grâce à des énoncés construits, pour une désignation autre que couteau.

Nous testerons cet force sur horloge dont « indiquer l'heure » est une propriété essentielle.

(i) Cette horloge ne donne plus l'heure (je vais la jeter)

(ii) Cette horloge exposée dans un musée ne donne plus l'heure (quelle intention?)

(iii)Cette horloge ne donne plus l'heure, mais c'est notre patrimoine (je ne vais pas la jeter)

Il y a dans horloge exposée dans un musée des stéréotypes qui atténuent une propriété essentielle de horloge. La qualification de «patrimonial »agit donc ici encore comme un modificateur déréalisant des stéréotypes de horloge. Il modifie l'orientation argumentative de l'énoncé. 
L'étude des représentations associées à la désignation d'un couteau en co(n)texte fonctionnel et patrimonial a montré que celles-ci évoluent, et que cette évolution agit non seulement sur le stéréotype du mot mais aussi sur ses propriétés essentielles, qui si elles ne disparaissent pas, sont abaisser. Elle a également montré que les traits du patrimoine « contaminent » la signification lexicale du mot, au point d'éventuellement modifier les orientations argumentatives que peut impliquer son emploi en contexte. Deux questions émergent de cette analyse : le caractère du cinétisme sémantique engendré par le cinétisme culturel qu'est la patrimonialisation sur les noms propres qui en font l'objet est-il systématique ? Par ailleurs ces transformations se font-elles toujours au profit des mêmes traits (symboliques) de patrimoine et aux dépens des mêmes types de composants sémantiques de l'objet concret? 


\section{BIBLIOGRAPHIE}

Anscombre, J.-C. et Ducrot, O. (1983), L'argumentation dans la langue, Bruxelles : P. Mardaga.

Anscombre, J.-C. (1995), Théorie des Topoï, Paris : Kimé.

Beghain, P. (1988), Le patrimoine : culture et lien social, Paris : Presses de Sciences Po. Bellachhab, A. et Galatanu, O. (2012), «La violence verbale : représentation sémantique, typologie et mécanismes discursifs », Signes, Discours et Sociétés, 9 [en ligne]. URL : www.revue-signes.info/document.php?id=2893.ISSN1338-8378

Bouchard, D., Evrard, I., Vocaj, E. (dir.) (2007), Représentation du sens linguistique, actes du colloque international de Montréal, 2003, Bruxelles : de Boeck, 313-325.

Choay, F. (2009), Le patrimoine en questions, anthologie pour un combat, Paris : Seuil.

Davallon, J. (2006), Le don du patrimoine, une approche communicationnelle de la patrimonialisation, Paris : Lavoisier.

Derrida, J. (1995), Mal d'archives : une impression freudienne, Paris : Galilée.

Desvallées, A. (2011), «Patrimoine » in Desvallées A, Mairesse F (eds) (2011), 427-452.

Ducrot, O. (1995), Les modificateurs déréalisants, Journal of Pragmatics 24 :145-165.

Gellereau, M. (2005), Les mises en scène de la visite guidée, Paris : L'Harmattan.

Galatanu, O. (1999), « Le phénomène sémantico-discursif de déconstructionreconstruction des topoï dans une sémantique argumentative intégrée » in Galatanu, O., Gouvrard, J.-M. (eds) (1999) : 41-51.

Galatanu, ,O. (2006), «Sémantique des possibles argumentatifs et dénomination » in Riegel, M., Schedecker, C., Swiggers, P. \& Tamba, I. (eds) (2006) : 499-510.

Galatanu, O. (2008), «L'interface linguistique-culturel et la construction du sens dans la communication didactique » in. Signes, discours et sociétés, 1 [en ligne] URL. http://www.revue-signes.info/document.php?id=263 ISSN1308-8378

Galatanu, O. (2009), « L'analyse du discours dans la perspective de la sémantique des possibles argumentatifs : les mécanismes sémantico-discursifs de construction du sens et de reconstruction de la signification lexicale », in Garric, N., Linghi, G. (eds) (2009), 49-68.

Kleiber, G. (1999), Problèmes de sémantique, la polysémie en questions, Lille : Presses universitaires du Septentrion.

Malraux, J. [1947] (1965), Le Musée imaginaire, Paris : Gallimard.

Mathieu, J., Léonidoff, (G.-P.) et Porter, J. (1987), «L'objet et ses contextes » in Bulletin d'histoire de la culture matérielle, $26: 7-18$.

Putnam, H. (1990), Représentation et réalité, Paris : Seuil. 


\section{DICTIONNAIRES}

Dictionnaire encyclopédique de muséologie (2011), Desvallées A., Mairesse F. (eds), Paris : A. Colin.

Dictionnaire de l'Académie française, classique Garnier [en ligne], [1694] (1785).

Nouveau dictionnaire de la langue française (1823), Paris : Laveaux.

Grand Robert de la langue française ( 2001), Paris : le Robert.

Dictionnaire Grand Larousse Universel [1984] (1994), Paris : Larousse.

Dictionnaire étymologique et historique du Français (1998), Paris : Larousse.

Dictionnaire Le Robert historique de la langue française (2012), Paris : le Robert.

Lexis, le Grand Littré (1975), Paris : Larousse.

Trésor de la langue française [en ligne] URL http://atilf.atilf.fr

SITOGRAPHIE COMPLÉMENTAIRE :

Www.auvergne-tourisme.info

www.encyclopedie.bourges.com 